\title{
Correlations of circulating miR-26b level with left ventricular hypertrophy and cardiac function in elderly patients with hypertension
}

\author{
Jian Fu', Fang Lin', \\ Zhengxia Pan ${ }^{3}$, Chun Wu
}

\begin{abstract}
Objectives: To study the correlations of circulating miR-26b level with left ventricular hypertrophy (LVH) and cardiac function in elderly patients with hypertension.

Methods: A total of 132 eligible patients were divided into low and high miR-26b level groups. Their baseline clinical data and biochemical indices were compared. The correlations between miR-26b level and echocardiographic parameters were studied by Pearson's analysis. Factors affecting LVH were explored by multivariate logistic regression analysis. The role of miR-26b in diagnosing LVH was predicted by receiver operating characteristic curve.

Results: The relative expression level of miR-26b was 4.56-16.93, with a median of 7.62. The two groups had similar baseline clinical data and biochemical indices $(P>0.05)$. Compared with high miR-26b level group, interventricular septal thickness (IVST), left ventricular posterior wall thickness (LVPWT), left ventricular mass index (LVMI) and number of LVH cases in low miR-26b level group significantly increased $(\mathrm{P}<0.05)$, and mitral ratio of peak early to late diastolic filling velocity $(E / A)$ decreased $(P<0.05)$. Circulating miR-26b level was negatively correlated with IVST, LVPWT and LVMI $(P<0.0001)$, and positively correlated with E/A $(\mathrm{P}<0.0001)$. The proportion of cardiac hypofunction cases in low miR-26b level group significantly exceeded that of high miR-26b level group $(P<0.05)$. Age and increased IVST, LVPWT and LVMI were independent risk factors for LVH $(P<0.05)$, and elevated miR-26b level was a protective factor $(P<0.05)$. AUC was 0.836 , and the optimal cutoff value was 8.83 , with high sensitivity and specificity.

Conclusions: MiR-26b level is negatively correlated with LVH and positively correlated with left ventricular diastolic function in elderly hypertensive patients. It is a protective factor for LVH complicated with diastolic dysfunction and a potential biomarker for diagnosis.
\end{abstract}

KEYWORDS: miR-26b; Elderly; Hypertension; Left ventricular hypertrophy; Cardiac function.

How to cite this:

doi: https://doi.org/10.12669/pjms.37.4.4048

Fu J, Lin F, Pan Z, Wu C. Correlations of circulating miR-26b level with left ventricular hypertrophy and cardiac function in elderly patients with hypertension. Pak J Med Sci. 2021;37(4):966-971. doi: https://doi.org/10.12669/pjms.37.4.4048

This is an Open Access article distributed under the terms of the Creative Commons Attribution License (http://creativecommons.org/licenses/by/3.0), which permits unrestricted use, distribution, and reproduction in any medium, provided the original work is properly cited.

Correspondence:

Zhengxia Pan

Department of Cardiac Surgery,

Children's Hospital of Chongqing Medical University,

National International Science and Technology Cooperation

Base for Children's Developmental Diseases,

Ministry of Education Key Laboratory of Child Development and

Disorders, Chongqing Key Laboratory of Pediatrics,

Chongqing 400014, P. R. China.

Email: 0626924@gmail.com

* Received for Publication:

* Accepted for Publication:
December 18, 2020

April 8, 2021

\section{INTRODUCTION}

As a common chronic disease bringing a serious economic burden, hypertension is mainly typified by the continuous rise of arterial blood pressure, and its long course changes the cardiac structure and function. The early manifestations of hypertension include left ventricular hypertrophy $(\mathrm{LVH})$ and diastolic dysfunction. As the disease progresses, ventricular systolic dysfunction and enlargement of the heart cavity occur, eventually 
triggering heart failure. ${ }^{1} \mathrm{LVH}$ is a common type of hypertension-induced target organ damage, mainly manifested as increased myocardial fibrosis. At present, its pathophysiological mechanism has not been fully clarified, but miRNAs have been verified to participate in cardiovascular pathophysiological processes such as heart development and remodeling, arrhythmia and myocardial hypertrophy. ${ }^{2}$ Circulating miRNAs, i.e. miRNAs in serum and plasma, can maintain extremely high stability under harsh conditions such as high temperature, acidity and alkalinity together with RNase. ${ }^{3}$ The correlations of changes in the circulating miR-26 level with $\mathrm{LVH}$ and cardiac function in elderly patients with hypertension have seldom been referred hitherto. In this study, therefore, the circulating miR-26b level and echocardiographic parameters in these patients were detected to explore their correlations, thereby providing some guidance for the early detection and diagnosis of LVH upon hypertension.

\section{METHODS}

A total of 132 elderly patients with hypertension treated in our hospital from January 2017 to October 2019 were selected. They were aged 6285 years old, including 80 males and 52 females. Hypertension was diagnosed based on the 2018 Chinese Guidelines for Prevention and Treatment of Hypertension ${ }^{4}$ : The blood pressures of patients taking no antihypertensive drugs were measured three times in different days, with diastolic blood pressure (DBP) of $\geq 90 \mathrm{mmHg}$ and/or systolic blood pressure (SBP) of $\geq 140 \mathrm{mmHg}$.

Inclusion criteria: 1) Patients aged over 60 years old; 2) patients who met the above diagnostic criteria for hypertension; 3) patients with primary hypertension; 4) patients who did not take antihypertensive drugs; 5) patients who signed the informed consent and actively cooperated in this study.

Exclusion criteria: 1) Patients with primary aldosteronism, pheochromocytoma, renal hypertension or other secondary hypertension; 2) patients with congenital cardiac malformation; 3) patients complicated with heart diseases such as hypertrophic cardiomyopathy, valvular heart disease and systolic heart failure; 4) patients with some diseases or those who had taken drugs affecting the heart rate; 5) patients with smoking, drinking or diabetes history; 6) patients complicated with severe liver, spleen, lung, kidney or other systemic diseases; 7) patients with metabolic diseases or tumors; 8) patients who received valve replacement, coronary artery bypass grafting, pacemaker implantation or surgery in the past year. This study was reviewed and approved by the Medical Ethics Committee of our hospital on January $6^{\text {th }} 2017$.

Baseline clinical data: The baseline clinical data of patients were collected, including gender, age, height and weight, and the body mass index (BMI) was calculated according to the following formula: BMI $=$ weight $(\mathrm{kg}) /[\text { height }(\mathrm{cm})]^{2}$. DBP and SBP of the patients measured by nurses in our hospital at admission were collected.

Detection of circulating miR-26b level: The venous blood was collected using a disposable vacuum blood collection tube containing anticoagulant ethylenediaminetetraacetic acid 12 hour after fasting in the morning of the next day after admission. Then the venous blood was let still for 1-2 hour at normal temperature and centrifuged at $4^{\circ} \mathrm{C}$ to obtain the plasma that was transferred into a sterilized RNase-free tube. Subsequently, total RNA was extracted from the plasma by the TRIzol method and quantified using a spectrophotometer. Next, the $3^{\prime}$ end of miRNAs was treated with poly(A), and reverse transcription was carried out. Using the synthesized cDNA as the template, RT-PCR was performed by the SYBR Green I method. Primers for miR-26b: F: 5'-ACACTCCAGCTGGGTTTGGTCCCCTTCAAC-3', R: 5'-GG TGTCGTGGAGTCGGCAATTCAGTTGAG-3' . Primers for U6: F: 5'-CTCGCTTCGGCAGCAC A-3', R: 5'-AACGCTTCACGAATTTGCGT-3'. The relative expression of miR-26b was calculated according to $2^{-\Delta \mathrm{Ct}}$.

Detection of biochemical indices: The venous blood was collected using a heparin sodium anticoagulation tube 12 hour after fasting in the morning of the next day after admission. Following centrifugation, the levels of fasting plasma glucose (FPG), total glyceride (TG), total cholesterol (TC), low-density lipoprotein cholesterol (LDL-C), high-density lipoprotein cholesterol (HDL-C), hemoglobin A1c (HbA1c), alanine aminotransferase (ALT), aspartate aminotransferase (AST), serum creatinine (Scr), creatine kinase (CK), creatine kinase-muscle/brain (CK-MB), lactate dehydrogenase (LDH), highsensitivity C-reactive protein (hs-CRP), blood urea nitrogen (BUN) and uric acid (UA) were measured with Olympus AU400 automatic biochemical analyzer (Japan). 
Echocardiography: Echocardiography was performed by the same ultrasonographer. According to the criteria recommended by the American Society of Echocardiography ${ }^{5}$, the left ventricular end diastolic diameter (LVEDD), interventricular septum thickness (IVST), left ventricular posterior wall thickness (LVPWT), left ventricular ejection fraction (LVEF\%), mitral valve early diastolic blood flow peak (E-Peak) and late diastolic flow peak (APeak) were measured by GE Vivid 7 color ultrasound system (USA) with a probe frequency of 2.5$3.5 \mathrm{MHz}$, and the ratio of E-Peak to A-Peak (E/A) was calculated. Three measured cardiac cycles were averaged. The left ventricular mass (LVM) was calculated based on the Devereux formula: $\mathrm{LVM}=1.04$ $\times\left[\left(\mathrm{LVEDD}^{2}+\mathrm{IVST}+\mathrm{LVPWT}^{3}-\mathrm{LVEDD}^{3}\right]-13.6\right.$. The left ventricular mass index (LVMI) was calculated by the body surface area (BSA) according to the formula below: LVMI $=$ LVM/BSA. LVMI of $>125 \mathrm{~g} /$ $\mathrm{m}^{2}$ (male) and $>110 \mathrm{~g} / \mathrm{m}^{2}$ (female) indicated LVH.

New York Heart Association (NYHA) classification: The patients were classified as per the standard formulated by NYH ${ }^{6}$ : Class I: There is no limitation of physical activity, and ordinary physical activity does not cause fatigue, dyspnea, palpitation, shortness of breath and angina pectoris symptoms. Class II: There is slight limitation of physical activity, and ordinary physical activity results in fatigue, dyspnea, palpitation, shortness of breath or angina pectoris symptoms, but patients are comfortable at rest. Class III: There is marked limitation of physical activity, and light physical activity causes dyspnea, palpitation or shortness of breath, but patients are comfortable at rest. Class IV: Patients are unable to carry on any physical activity, and dyspnea, palpitation, shortness of breath, angina pectoris or cardiac insufficiency occurs at rest. Patients in class I had normal cardiac function, while those in class II-IV suffered from cardiac dysfunction.

Statistical analysis: All data were statistically analyzed by SPSS 22.0 software. The numerical data were expressed as percentage [n (\%)] and subjected to the $\chi^{2}$ test. The quantitative data were represented as 'mean \pm standard deviation, and intergroup comparisons were conducted by the independent $\mathrm{t}$ test. The correlations between miR-26b level and various echocardiographic parameters were studied by the Pearson's analysis. Multivariate logistic regression analysis was carried out to explore the factors affecting $\mathrm{LVH}$. The role of circulating miR$26 \mathrm{~b}$ in diagnosing $\mathrm{LVH}$ was predicted by plotting the receiver operating characteristic (ROC) curve. $\mathrm{P}<0.05$ was considered statistically significant.

\section{RESULTS}

Baseline clinical data of patients with different miR-26b levels: The relative expression level of miR-26b in 132 patients was 4.56-16.93, with a median of 7.62 , so patients with miR-26b levels of $<7.62$ and $\geq 7.62$ were included into low and high miR-26b level groups, respectively. The two groups had similar baseline clinical data including gender, age, BMI, DBP and SBP as well as biochemical indices such as FPG, TG, TC, LDL-C, HDL-C, HbA1c, ALT, AST, Scr, CK, CK-MB, LDH, hs-CRP, BUN and UA (P>0.05) (Table-I).

Compared with high miR-26b level group, IVST, LVPWT, LVMI and number of LVH cases in low miR-26b level group significantly increased $(\mathrm{P}<0.05)$, and the E/A ratio significantly decreased $(\mathrm{P}<0.05)$. The two groups had similar LVEDD and LVEF (P>0.05) (Table-II).

The circulating miR-26b level was significantly negatively correlated with IVST, LVPWT and LVMI

Table-I: Baseline clinical data of patients with different miR-26b levels [case $(\%)](\bar{X} \pm \mathrm{s})$.

\begin{tabular}{|c|c|c|c|c|}
\hline Item & $\begin{array}{l}\text { Low miR- } 26 b \\
\text { level group } \\
(n=76)\end{array}$ & $\begin{array}{l}\text { High miR-26b } \\
\text { level group } \\
(n=56)\end{array}$ & $x^{2} / t$ & $P$ \\
\hline Gender (case) & & & 1.217 & 0.269 \\
\hline Male & 43 & 37 & & \\
\hline Female & 33 & 19 & & \\
\hline Age (year) & $71.85 \pm 7.03$ & $72.16 \pm 7.09$ & 0.249 & 0.803 \\
\hline BMI $\left(\mathrm{kg} / \mathrm{cm}^{2}\right)$ & $25.41 \pm 3.28$ & $24.92 \pm 3.37$ & 0.838 & 0.403 \\
\hline DBP (mmHg) & $93.52 \pm 8.24$ & $92.87 \pm 8.15$ & 0.450 & 0.653 \\
\hline SBP (mmHg) & $148.93 \pm 13.14$ & $150.12 \pm 13.78$ & 0.504 & 0.615 \\
\hline FPG (mmol/L) & $5.57 \pm 0.52$ & $5.63 \pm 0.59$ & 0.619 & 0.537 \\
\hline TG (mmol/L) & $2.41 \pm 0.58$ & $2.42 \pm 0.55$ & 0.100 & 0.920 \\
\hline TC (mmol/L) & $5.39 \pm 0.65$ & $5.36 \pm 0.62$ & 0.267 & 0.789 \\
\hline LDL-C (mmol/L & $2.62 \pm 0.31$ & $2.59 \pm 0.28$ & 0.572 & 0.568 \\
\hline HDL-C (mmol/I & L) $1.35 \pm 0.34$ & $1.32 \pm 0.31$ & 0.519 & 0.604 \\
\hline HBA1c (\%) & $5.18 \pm 0.67$ & $5.24 \pm 0.69$ & 0.502 & 0.616 \\
\hline $\operatorname{ALT}(\mathrm{U} / \mathrm{L})$ & $30.21 \pm 9.93$ & $29.85 \pm 9.74$ & 0.208 & 0.836 \\
\hline AST (U/L) & $27.56 \pm 8.24$ & $28.16 \pm 8.82$ & 0.401 & 0.689 \\
\hline Scr $(\mu \mathrm{mol} / \mathrm{L})$ & $75.94 \pm 13.79$ & $77.01 \pm 14.35$ & 0.433 & 0.666 \\
\hline CK (U/L) & $96.48 \pm 22.56$ & $95.39 \pm 21.78$ & 0.278 & 0.781 \\
\hline CK-MB (U/L) & $16.13 \pm 4.89$ & $14.91 \pm 4.04$ & 1.523 & 0.130 \\
\hline LDH (U/L) & $144.76 \pm 31.25$ & $141.28 \pm 30.97$ & 0.635 & 0.527 \\
\hline HsCRP (mg/L) & $12.37 \pm 5.68$ & $11.94 \pm 5.26$ & 0.443 & 0.658 \\
\hline $\mathrm{BUN}(\mathrm{mmol} / \mathrm{L})$ & $5.41 \pm 1.39$ & $5.37 \pm 1.34$ & 0.166 & 0.868 \\
\hline $\mathrm{UA}(\mu \mathrm{mol} / \mathrm{L})$ & $385.42 \pm 39.76$ & $392.23 \pm 40.15$ & 0.969 & 0.335 \\
\hline
\end{tabular}


Table-II: Echocardiographic parameters of patients with different miR-26b levels [case $(\%)](\bar{X} \pm \mathrm{s})$.

\begin{tabular}{lcccc}
\hline Item & $\begin{array}{c}\text { Low miR-26b } \\
\text { level group } \\
(n=76)\end{array}$ & $\begin{array}{c}\text { High miR-26b } \\
\text { level group } \\
(n=56)\end{array}$ & $\chi^{2} / t$ & $P$ \\
\hline LVEDD (mm) & $44.57 \pm 3.94$ & $44.25 \pm 3.86$ & 0.465 & 0.643 \\
IVST (mm) & $12.23 \pm 1.17^{*}$ & $9.96 \pm 0.92$ & 12.031 & 0.000 \\
LVPWT (mm) & $11.69 \pm 1.04^{*}$ & $9.57 \pm 0.95$ & 12.003 & 0.000 \\
LVEF (\%) & $65.72 \pm 6.48$ & $66.13 \pm 6.54$ & 0.358 & 0.721 \\
E/A & $0.59 \pm 0.14^{*}$ & $0.92 \pm 0.31$ & 8.219 & 0.000 \\
LVMI (g/m2) & $139.35 \pm 14.25^{*}$ & $110.42 \pm 10.78$ & 12.738 & 0.000 \\
LVH (case, \%) & $70(92.11)^{*}$ & $13(23.21)$ & 65.558 & 0.000 \\
\hline Compared with
\end{tabular}

Compared with high miR-26b level group, ${ }^{*} \mathrm{P}<0.05$.

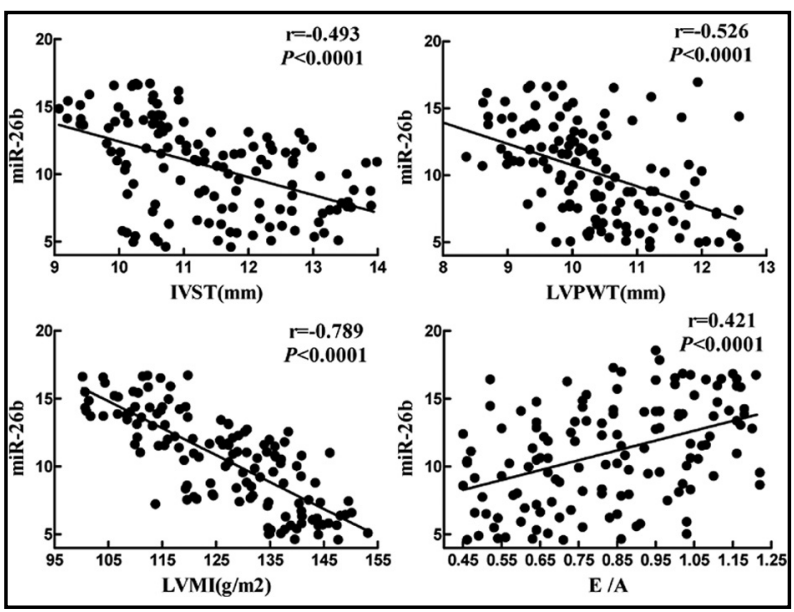

Fig.1: Correlations of miR-26b level with IVST, LVPWT, LVMI and E/A ratio.

$(\mathrm{r}=-0.493,-0.526,-0.789, \mathrm{P}<0.0001)$, and positively correlated with E/A ( $\mathrm{r}=0.421, \mathrm{P}<0.0001)$ (Fig.1).

The proportion of cardiac hypofunction cases in low miR-26b level group $(72 / 76,94.74 \%)$ was significantly exceeded that of high miR-26b level group $(26 / 56,46.43 \%)(\mathrm{P}<0.05)$ (Table-III).

Table-III: NYHA classification of patients with different miR-26b levels [case (\%)].

\begin{tabular}{lcccc}
\hline Item & $\begin{array}{c}\text { Low miR-26b } \\
\text { level group } \\
(n=76)\end{array}$ & $\begin{array}{c}\text { High miR-26b } \\
\text { level group } \\
(n=56)\end{array}$ & $\chi^{2}$ & $P$ \\
\hline Class I (case, \%) & $4(5.26)^{*}$ & $33(58.93)$ & & \\
Class II (case, \%) & $32(42.11)^{*}$ & $19(33.93)$ & & \\
Class III (case, \%) & $30(39.47)^{*}$ & $3(5.36)$ & & \\
Class IV (case, \%) & $10(13.16)^{*}$ & $1(1.79)$ & \\
Cardiac & $72(94.74)^{*}$ & $26(46.43)$ & 42.861 & 0.000 \\
hypofunction (case, \%) & & & \\
\hline Compared with high miR-26b level group, *P<0.05.
\end{tabular}

Compared with high miR-26b level group, ${ }^{*} \mathrm{P}<0.05$.
Table-IV: Factors affecting LVH in elderly patients with hypertension.

\begin{tabular}{lccccc}
\hline Factor & $\beta$ & $S E$ & Wald & $P$ & OR $(95 \% C I)$ \\
\hline Age & 1.734 & 0.927 & 3.419 & 0.021 & $2.379(1.368 \sim 3.842)$ \\
IVST & 1.295 & 0.646 & 2.358 & 0.013 & $5.142(3.405 \sim 6.257)$ \\
LVPWT & 0.867 & 0.719 & 6.642 & 0.032 & $3.063(2.241 \sim 5.463)$ \\
LVMI & 2.453 & 0.882 & 4.734 & 0.006 & $4.581(3.263 \sim 7.879)$ \\
miR-26b & -2.246 & 0.954 & 7.527 & 0.000 & $0.528(0.257 \sim 0.895)$ \\
\hline
\end{tabular}

Age: $>75$ years old $=1, \leq 75$ years old $=0$; IVST: increase $=1$, no increase $=0$; LVPWT: increase $=1$, no increase $=0$; LVMI: male $>125 \mathrm{~g} / \mathrm{m} 2$, female $>110 \mathrm{~g} / \mathrm{m} 2=1$, male $\leq 125 \mathrm{~g} / \mathrm{m} 2$, female $\leq 110 \mathrm{~g} / \mathrm{m} 2=0 ;$ miR-26b: $\geq 7.62=1,<7.62=0$.

Age and increased IVST, LVPWT and LVMI were independent risk factors for $\mathrm{LVH}$ in elderly patients with hypertension $(\mathrm{P}<0.05)$, and elevated miR-26b level was a protective factor $(\mathrm{P}<0.05)$ (Table-IV).

The area under ROC curve was 0.836, with $95 \% \mathrm{CI}$ of $0.757 \sim 0.916(\mathrm{P}<0.05)$. The optimal cutoff value was 8.83 , the sensitivity was $81.4 \%$ and the specificity was $78.9 \%$. Therefore, miR-26b level had high diagnostic value for LVH in elderly patients with hypertension (Fig.2).

\section{DISCUSSION}

$\mathrm{LVH}$ is one of the common complications of hypertension. Long-term hypertension can increase the left ventricular afterload of the heart and thus leads to this chronic adaptive change, i.e. the occurrence of LVH. ${ }^{7}$ The rate of arrhythmia in patients with hypertension and LVH was

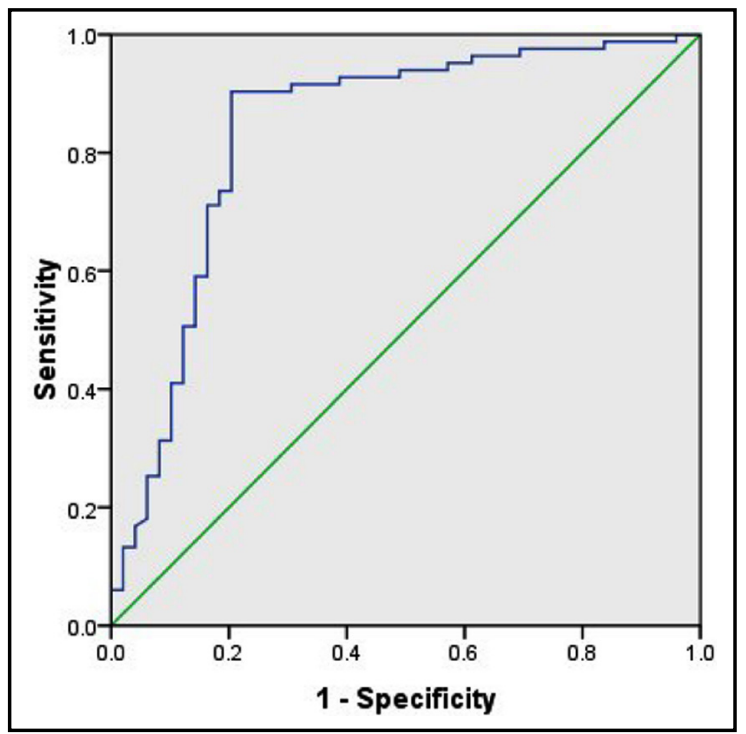

Fig.2: ROC curve of miR-26b level for $\mathrm{LVH}$ in elderly patients with hypertension. 
significantly higher than that in patients with simple hypertension. ${ }^{8}$ The coronary reserve capacity of patients with hypertension and LVH is significantly reduced, resulting in acute myocardial ischemic events that easily occur and increase the risk of death. ${ }^{9}$ At present, the commonly used diagnostic methods such as electrocardiogram, magnetic resonance imaging and echocardiography have disadvantages or limitations. ${ }^{10}$ There is an urgent need to find a simple, specific and highly sensitive diagnostic method in clinical practice.

As endogenous non-coding small-molecule RNAs that are highly conserved during biological evolution, miRNAs can regulate gene expression at the post-transcriptional level. ${ }^{11}$ The roles of miRNAsindiseases suchas viraldiseases, digestive system, nervous system and reproductive system are well-documented. ${ }^{12}$ They have been involved in cardiac development and remodeling, and are closely related to various cardiovascular diseases such as myocardial fibrosis, myocardial hypertrophy and heart failure. ${ }^{13}$ The expression level of miR-26b in patients with coronary heart disease is significantly lower than that in healthy people, and miR-26b is expected to become a marker for the diagnosis of coronary heart disease. ${ }^{14}$ In a mouse model of atrial fibrosis and fibrillation, miR-26 inhibits the Ang II/KLF 4/ TGF- $\beta$ signaling pathway activation, thereby combating atrial fibrillation and atrial fibrosis. ${ }^{15}$ In this study, the miR-26b levels in the venous blood of 132 elderly hypertensive patients were detected. The results showed that the relative expression level was between 4.56 and 16.93, and the median was 7.62 as the group cut-off value, and the value of $<7.62$ was recorded as miR-26b low level group, $\geq 7.62$ is recorded as miR-26b high level group. The two groups had similar baseline clinical data including gender, age, BMI, DBP and SBP as well as biochemical indices such as FPG, TG, TC, LDL-C, HDL-C, HbA1c, ALT, AST, Scr, CK, CK-MB, LDH, hs-CRP, BUN and UA $(\mathrm{P}>0.05)$.

Compared with high miR-26b level group, IVST, LVPWT, LVMI and number of LVH cases in low miR-26b level group significantly increased $(\mathrm{P}<0.05)$. IVST and LVPWT determine LVMI, and the left ventricular weight index can be directly used as an important clinical index to measure LVH. A high LVMI means more serious $\mathrm{LVH} .{ }^{16}$ Thus, the lower the circulating miR-26b level, the more likely LVH occurs. Besides, an E/A ratio of $<1$ suggests decline of the left ventricular diastolic function. ${ }^{17}$ Elderly hypertensive patients with LVH often suffer from decreased left ventricular diastolic function. ${ }^{18}$ This study showed that the E/A ratio of both groups was $<1$, and the value of the miR-26b low level group was significantly lower than that of the miR-26b high level group $(\mathrm{P}<0.05)$. Accordingly, both groups of had left ventricular diastolic dysfunction, and the lower the miR-26b level, the more serious. Moreover, the circulating miR-26b level was significantly negatively correlated with IVST, LVPWT and LVMI $(\mathrm{P}<0.0001)$, and positively correlated with E/A $(\mathrm{P}<0.0001)$. The proportion of cardiac hypofunction cases in low miR-26b level group significantly exceeded that of high miR$26 \mathrm{~b}$ level group $(\mathrm{P}<0.05)$. Collectively, elderly hypertensive patients with low miR-26b levels were more likely to have LVH and cardiac hypofunction.

Multivariate analysis showed that age and increased IVST, LVPWT and LVMI were independent risk factors for LVH in elderly patients with hypertension $(\mathrm{P}<0.05)$, and elevated miR$26 \mathrm{~b}$ level was a protective factor $(\mathrm{P}<0.05)$. With increasing age, the volume of myocardial cells increases, the heart collagen component and heart weight increase, long-term overload pressure and left ventricular adaptive reaction failure may easily lead to $\mathrm{LVH} .{ }^{19}$ The expression of miR-26a and miR$26 \mathrm{~b}$ is down-regulated in rat cardiac hypertrophy tissues, and inhibiting the target glycogen synthase kinase- $3 \beta$ can regulate the cardiac hypertrophy process. ${ }^{20}$ Herein, miR-26b also played a regulatory role in the process of $\mathrm{LVH}$ in elderly hypertensive patients, but its mechanism remains elusive. ROC curve analysis showed that the area under the curve was $0.836,95 \% \mathrm{CI}$ was $0.757 \sim 0.916(\mathrm{P}<0.05)$, the optimal cutoff value was 8.83 , the sensitivity was $81.4 \%$, and the specificity was $78.9 \%$, suggesting that the miR-26b expression level of $<8.83$ can be diagnosed as LVH sensitively and specifically.

\section{CONCLUSION}

In summary, the circulating miR-26b level is negatively correlated with LVH and positively correlated with left ventricular diastolic function in elderly patients with hypertension. It is a protective factor for $\mathrm{LVH}$ complicated with diastolic dysfunction and a potential biomarker for the diagnosis of LVH in these patients.

Declaration of interest: The author declares no competing interest.

Source of funding: This study was not financially supported. 


\section{REFERENCES}

1. Theilen LH, Meeks H, Fraser A, Esplin MS, Smith KR, Varner MW. Long-term mortality risk and life expectancy following recurrent hypertensive disease of pregnancy. Am J Obstet Gynecol. 2018;219(1):107.e1-6. doi: 10.1016/j. ajog.2018.04.002

2. Wu QS, He Q, He JQ, Chao J, Wang WY, Zhou Y, et al. The role of mitofilin in left ventricular hypertrophy in hemodialysis patients. Renal Fail. 2018;40(1):252-258. doi: 10.1080/0886022X.2018.1456455

3. Sohel MH. Extracellular/circulating microRNAs: release mechanisms, functions and challenges. Achievement Life Sci. 2016;10(2):175-186. doi: 10.1016/j.als.2016.11.007

4. Joint Committee for Guideline Revision, Chinese Hypertension League, Chinese Society of Cardiology, Hypertension Committee of the Chinese Medical Doctor Association. [2018 Chinese Guidelines for Prevention and Treatment of Hypertension]. Chin J Cardiovasc Med. 2019;24:24-56.

5. Xu CF, Tan ES, Feng L, Santhanakrishnan R, Chan MM, Nyunt SZ, et al. Electrocardiographic criteria for left ventricular hypertrophy in Asians differs from criteria derived from Western populations-community-based data from an Asian population. Ann Acad Med Singap. 2015;44(8):274-283.

6. Vallee A, Yannoutsos A, Temmar M, Tubiana CD, Spinu I, Zhang Y, et al. Determinants of the aortic pulse wave velocity index in hypertensive and diabetic patients: predictive and therapeutic implications. J Hypertens. 2018;36(12):2324-2332. doi: 10.1097/HJH.0000000000001828

7. Kühl JT, Nielsen JB, Stisen ZR, Fuchs A, Sigvardsen PE, Graff $C$, et al. Left ventricular hypertrophy identified by cardiac computed tomography and ECG in hypertensive individuals: a population-based study. J Hypertens. 2018;37(4):739-746. doi: 10.1097/HJH.0000000000001962

8. Silva CT, Zorkoltseva IV, Niemeijer MN, Van Den Berg ME, Amin N, Demirkan A, et al. A combined linkage, microarray and exome analysis suggests MAP3K11 as a candidate gene for left ventricular hypertrophy. BMC Medi Genomics. 2018;11(1):22. doi: 10.1186/s12920-018-0339-9.

9. Cao JL, Yang YQ, Nabeel DM, Sun YL, Kong XQ, Lu XZ. Correlation between Serum Calcineurin Activity and Left Ventricular Hypertrophy in Hypertensive Patients and Its Clinical Significance. Cardiology. 2018;139(2):124-131. doi: 10.1159/000481280

10. Sun N, Chen JW, Wang J, Xie L, Chen L, Mu J, et al. Asian expert consensus for the diagnosis and treatment of hypertension-associated left ventricular hypertrophy. Cardiol Plus. 2016;1(3):37-47. doi: 10.4103/2470-7511.248356

11. Katz MG, Fargnoli AS, Williams RD, Kendle AP, Steuerwald NM, Bridges CR. miRNAs as potential molecular targets in heart failure. Future Cardiol. 2014;10(6):789-800. doi: $10.2217 /$ fca. 14.64

12. Shen J, Xing W, Gong F, Wang W, Yan Y, Zhang Y, et al. MiR-150-5p retards the progression of myocardial fibrosis by targeting EGR1. Cell Cycle. 2019;18(12):1335-1348. doi: 10.1080/15384101.2019.1617614

13. Tomaniak M, Sygitowicz G, Błaszczyk O, Kołtowski L, Puchta D, Malesa K, et al. MiR-1, miR-21, and galectin-3 in hypertensive patients with symptomatic heart failure and left ventricular hypertrophy. Kardiol Pol. 2018;76(6):1009 1011. doi: $10.5603 /$ KP.2018.0117
14. Hinkel R, Ng JK, Kupatt C. Targeting microRNAs for cardiovascular therapeutics in coronary artery disease. Curr Opin Cardiol. 2014;29(6):586-594. doi: 10.1097/ HCO.0000000000000107

15. Zhao X, Xia SJ, Sun PY. [miR-26 inhibit atrial fibrillation susceptibility and atrial fibrosis via targeting the Ang II/ KLF4/TGF- $\beta$ pathway]. J Clin Cardiol. 2019;35:549-554.

16. Antikainen RL, Peters R, Beckett NS, Fagard RH, Wang JG, Rajkumar C, et al. Left ventricular hypertrophy is a predictor of cardiovascular events in elderly hypertensive patients: Hypertension in the Very Elderly Trial. J Hypertens. 2016;34(11):2280-2286. doi: 10.1097/HJH.0000000000001073

17. Jakob P, Kacprowski T, Briand-Schumacher S, Heg D, Klingenberg R, Stahli BE, et al. Profiling and validation of circulating microRNAs for cardiovascular events in patients presenting with ST-segment elevation myocardial infarction. Eur Heart J. 2017;38(7):511-515. doi: 10.1093/ eurheartj/ehw563

18. Chowdhury EK, Jennings GL, Dewar E, Wing LM, Reid CM. Predictive Performance of Echocardiographic Parameters for Cardiovascular Events Among Elderly Treated Hypertensive Patients. Am J Hypertens. 2016;29(7):821-831. doi: 10.1093/ajh/hpw035

19. Restrepo C, Patel SK, Rethnam V, Werden E, Ramchand J, Churilov L, et al. Left ventricular hypertrophy and cognitive function: a systematic review. J Hum Hypertens. 2018;32(3):171-179. doi: 10.1038/s41371-017-0023-0

20. Vegter EL, Ovchinnikova ES, Silljé HHW, Meems LM, van der Pol A, van der Velde AR, et al. Rodent heart failure models do not reflect the human circulating microRNA signature in heart failure. PLoS One. 2017;12(5):e0177242. doi: 10.1371/journal.pone.0177242

\section{Authors' Contributions:}

JF \& ZXP Designed this study and significantly revised this manuscript.

FL Drafted this manuscript.

FL \& CW Collected and analyzed clinical data.

All authors agree to the submission and publication of this manuscript.

Authors:

1. Jian Fu,

2. Fang Lin,

The Second Affiliated Hospital of The Army Medical University, No. 136, Zhongshan Second Road,

Yuzhong District,

Chongqing 400014, P.R. China.

3. Zhengxia Pan,

4. Chun Wu

1,3,4: Department of Cardiac Surgery,

Children's Hospital of Chongqing Medical University, National International Science and Technology Cooperation Base for Children's Developmental Diseases,

Ministry of Education Key Laboratory of Child Development and Disorders, Chongqing Key Laboratory of Pediatrics, Chongqing 400014,

P.R. China. 